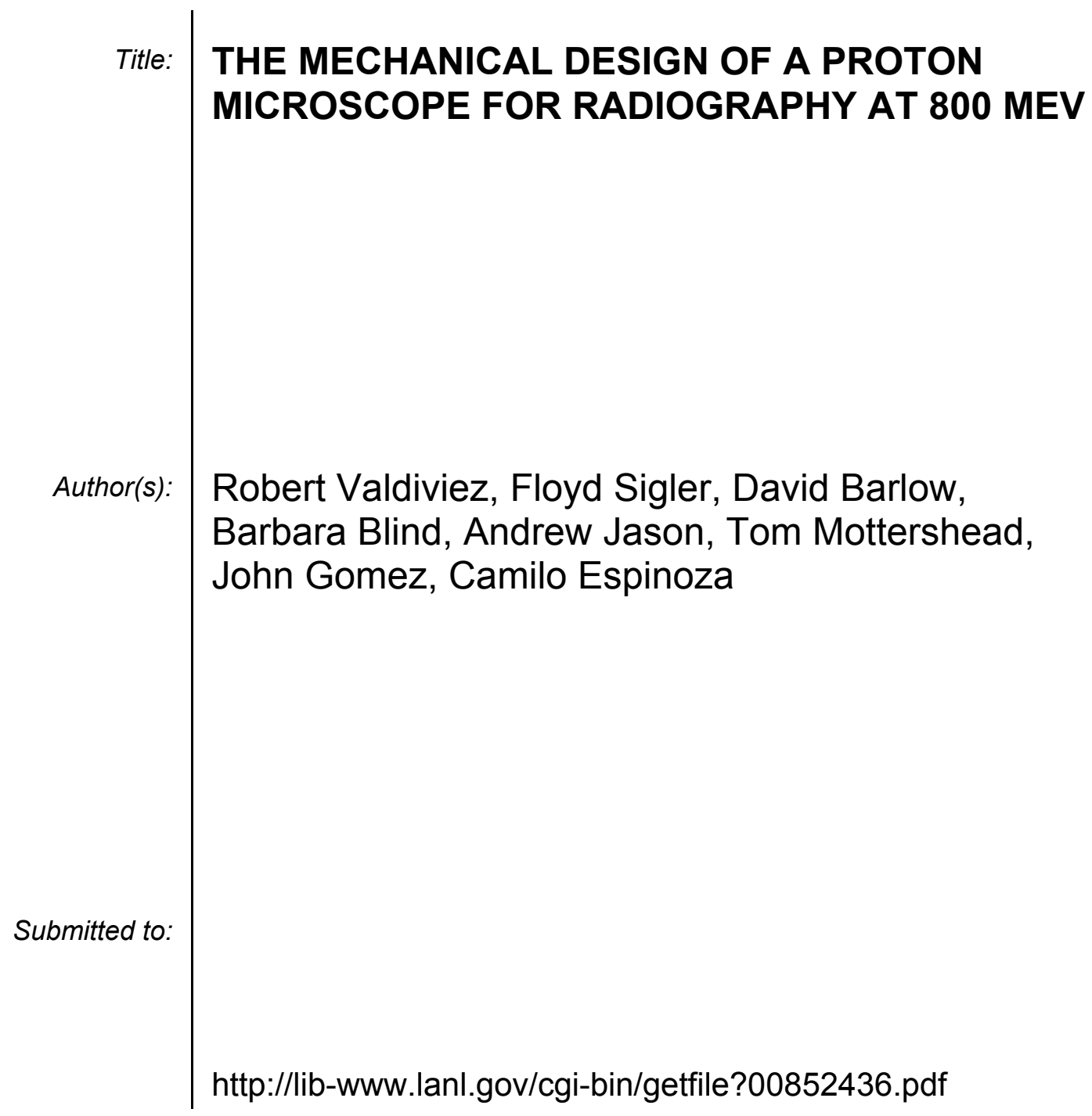
Laboratory requests that the publisher identify this article as work performed under the auspices of the U.S. Department of Energy. Los Alamos National Laboratory strongly supports academic freedom and a researcher's right to publish; as an institution, however, the Laboratory does not endorse the viewpoint of a publication or guarantee its technical correctness. 


\title{
THE MECHANICAL DESIGN OF A PROTON MICROSCOPE FOR RADIOGRAPHY AT $800 \mathrm{MEV}^{1}$
}

\author{
Robert Valdiviez, Floyd Sigler, David Barlow, Barbara Blind, Andrew Jason, Tom Mottershead, \\ John Gomez, Camilo Espinoza \\ Los Alamos National Laboratory
}

\section{Abstract}

A proton microscope has been developed for radiography applications using the $800-\mathrm{MeV}$ linear accelerator at the Los Alamos Neutron Science Center (LANSCE). The microscope provides a magnified image of a static device, or of a dynamic event such as a high-speed projectile impacting a target. The microscope assembly consists primarily of four Permanent Magnet Quadrupoles (PMQ's) that are supported on movable platforms. The platform supports, along with the rest of the support structure, are designed to withstand the residual dynamic loads that are expected from the dynamic tests. This paper covers the mechanical design of the microscope assembly, including the remote positioning system that allows for fine-tuning the focus of an object being imaged.

\section{INTRODUCTION}

Figure 1 shows the microscope assembly installed on the beam line in Area $\mathrm{C}$ of LANSCE. The beam direction is left to right. Area $\mathrm{C}$ is the experimental area where proton radiography is performed on a variety of experiment types. The experiments that have been imaged range from the shock propagation through high-explosive configurations to the flow pattern of liquids through coolant channels in industrial equipment.

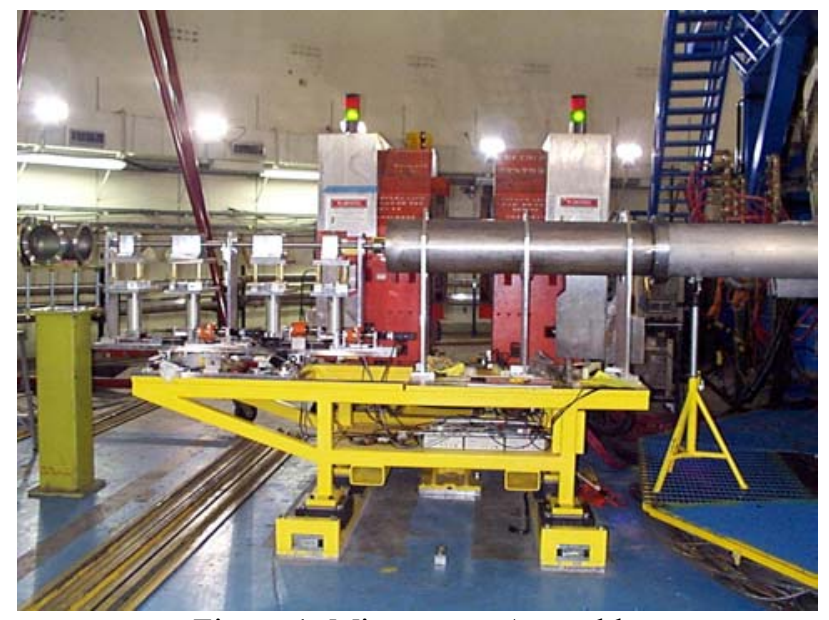

Figure 1: Microscope Assembly
The magnetic lattice of the proton radiography beam-line consists mainly of two magnetic sections [1]. The first section is an illumination section that is up-stream of the object or event being imaged, contains dipole and quadrupole magnets along with beam diagnostic assemblies, and provides for beam conditioning for the imaging task at hand. The second section is a focusing section that consists of four nominally 12 -inch-diameter bore quadrupole magnets and a beam collimator. The focusing section provides for transport of the scattered proton beam to the imaging plane. A second focusing section and imaging plane that is located down-stream of the first focusing section is available if necessary for a given imaging experiment.

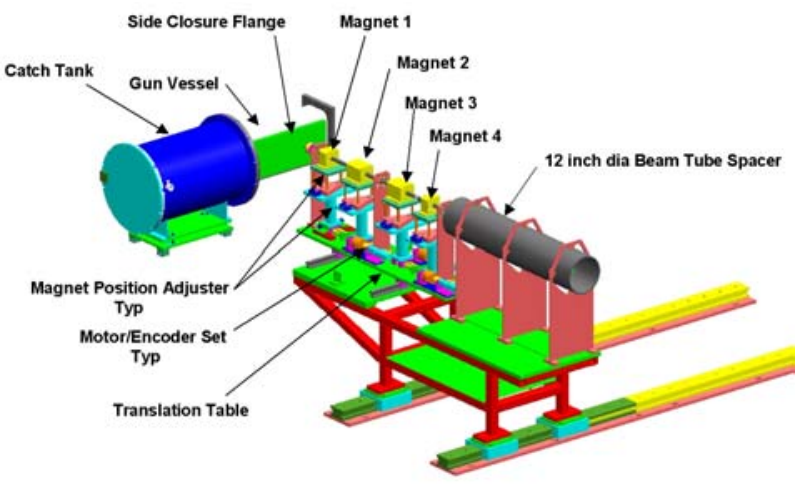

Figure 2: Microscope Assembly

The proton microscope assembly and PMQ lattice [2], shown in Figure 2, replaces the first focusing section of the proton radiography lattice. Two of the large electromagnetic quadrupole magnets are physically rolled off of the beam line via tracks on the floor, and the microscope assembly is rolled into their place for magnified imaging tasks. The other two electromagnetic quadrupole magnets remain in place, but are not powered. In this magnified imaging configuration the four PMQ's of the microscope assembly provide all of the down-stream beam focusing. An image magnification of approximately seven times is possible with the microscope. A small beam collimator is contained in the beam tube of the microscope assembly for controlling the image contrast.

\footnotetext{
${ }^{1}$ This work sponsored by the U.S. Department of Energy, NNSA
} 


\section{THE MECHANICAL DESIGN OF THE PROTON MICROSCOPE}

The microscope assembly consists of a main support structure, a transition beam tube that consists of a 1.25-inch-diameter and a 12-inch-diameter tube that are joined in series, a magnet lattice support plate that provides for lateral translation of the entire magnetic lattice off of the beam-line, the four individual magnet support bases, and the four sets of PMQ's. Remote positioning of three of the PMQ sets is also included in the design. The three down-stream PMQ sets can have their longitudinal, or $\mathrm{Z}$ position varied over approximately $+/-3.0$ inches to allow for focusing adjustments. This positioning is done by using motor and encoder sets that communicate to a Personal Computer (PC) in the counting house for the experimental area. The motor and encoder sets move the support bases of the PMQ's.

The main support structure of the microscope is a welded structural steel frame, and is shown in Figure 3.

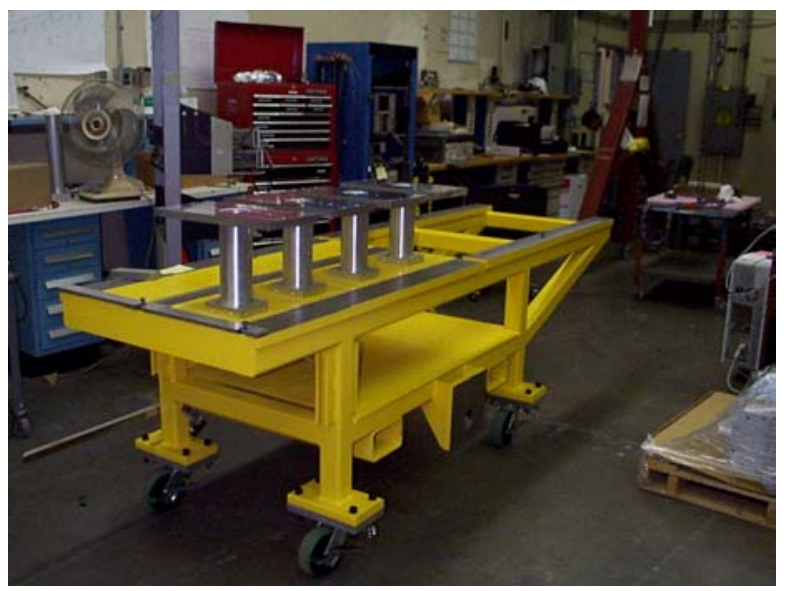

Figure 3: Microscope Assembly Support Stand

The structure is designed to resist the residual shock loads that it can experience from dynamic tests. Dynamic tests that the microscope would image would be limited to small high-explosive-mass quantities, thus producing low residual dynamic loads on the support structure. However, since the microscope could be parked in storage near much higher energetic tests being conducted, the design considered higher shock loadings, in the range of $2 \mathrm{~g}$, to prevent inadvertent damage to the microscope. The support frame is also designed to resist seismic loads for personnel safety considerations. The support structure also has its center of gravity biased to the low center region using thick steel plates. This center of gravity design has been used to provide static stability regardless of the microscope's configuration. Magnets and beam tubes can be removed without producing a tipping hazard. The weight of the support structure is 2377 pounds. The total weight of the microscope assembly is 3400 pounds.
The transition beam tube allows for coupling into the gun or test vessel on one end, and the existing lattice beam tube on the downstream end. The 1.25 -inch-diameter tube, with a 0.12 -inch-wall thickness, couples into the test vessel. Since high-speed material impact tests (velocities of approximately $6100 \mathrm{feet} / \mathrm{sec}$ ) are conducted in the vessel the adjoining beam tube has a thick wall to resist penetration damage from shrapnel of the impact experiment. The 1.25-inch diameter beam tube can be removed and replaced without having to disassemble the microscope assembly. The 1.25-inch-diameter tube couples into the 12-inch-diameter tube using a flange and a transition piece. The 12-inch-diameter tube has a 0.5 -inch-thick wall.

The magnet support bases are mounted on a main support plate. This support plate is mounted on rails that are aligned laterally to the beam line. The main support plate translates the four magnet sets, support bases, and 1.25 -inch diameter beam tube off of the beam line. This feature is included to allow for quick replacement of a beam tube. Beam tube replacement will be necessary when the tube inside surface has significant damage done to it, or when the beam collimator is to be changed in a given imaging set-up. The beam collimator is integral to the beam tube.

The magnet support bases provide for the support of the PMQ sets, and their alignment. Figure 4 shows two PMQ sets and their respective support bases. Each PMQ set support base provides five degrees of freedom for magnet alignment. The sixth degree of freedom, longitudinal, or Z, position is provided manually for the first PMQ set, and remotely for the other three.

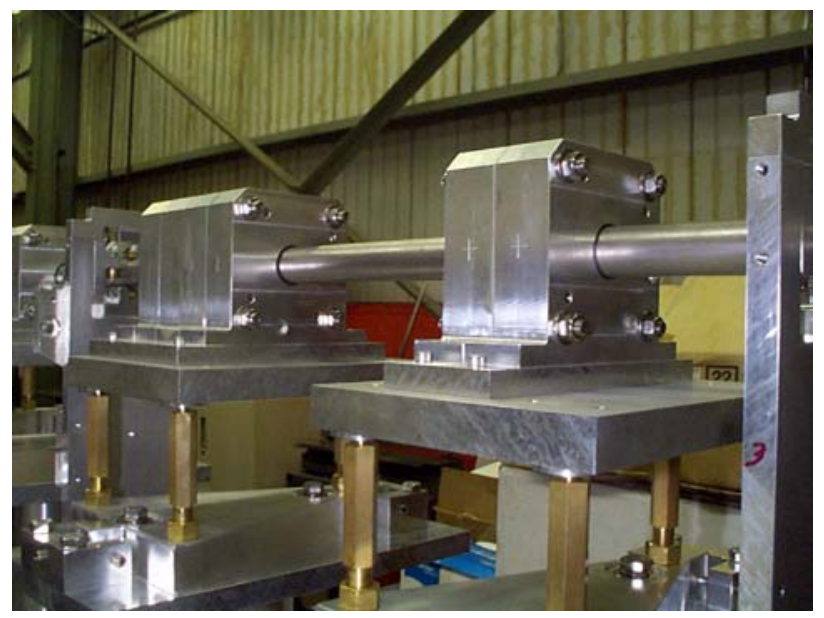

Figure 4: PMQ's and Support Bases

The PMQ sets are made by combining single PMQs into a set of two or four. A single PMQ has an average measured quadrupole gradient-length (GL) product of approximately $2.81 \mathrm{~T}$. By combining two or four PMQ's the necessary magnet strength is obtained for each set. Each PMQ has an inside bore of 1.375 inch, leaving a nominal gap of 0.062 inch between the magnet bore and 
the outside of the 1.25-inch diameter beam tube. The gap is needed to prevent the beam tube from impacting the magnet bore during dynamic testing.

\section{ASSEMBLY OF THE PMQ SETS}

The PMQ sets are assembled by separating the individual PMQ's beyond their field-influence distance on a rail assembly. Alignment rods are placed through two of the clamping holes in the PMQ bodies, and a clamping assembly is used to draw the magnets together. At a separation distance of approximately 0.008 inch the repulsive force between two PMQ's is approximately 350 pounds. The PMQ's are drawn together to where no axial gaps are present, permanent alignment rods and bolts installed and tightened, and the PMQ set is then removed from the clamping fixture. The PMQ set is then ready for installation onto the microscope assembly.

\section{THE MAGNET MOTION CONTROL SYSTEM}

The remote-motion control for the three down-stream PMQ sets of the microscope is accomplished by having each of the magnet support bases mounted on one common rail. A motor-encoder set is used on each magnet support base to change its longitudinal, or $\mathrm{Z}$, position. The support bases are held in place with the use of an electrically deactivated brake that is an integral part of the motor assembly. A clutch assembly is also placed in-line with the drive shaft. If the drive components meet too much drive resistance, then the clutch slips and prevents damage to any of the components.

The motors communicate with the control $\mathrm{PC}$ via the RS232 communication protocol. The encoders communicate in the RS485 communication protocol. The encoder RS485 signal is converted to the RS232 protocol via an in-line converter device. All signals are converted from the RS232 protocol to a fiber optic signal for the transmission length between the PC in the counting house and the experimental area. A fiber-optic signal is used for the majority of the control transmission length of 425 feet in order to eliminate possible electrical interference from other cabling.

A user interface program on the $\mathrm{PC}$ enables the microscope user to query the encoders to view their current $\mathrm{Z}$ positions, and make any positional changes. The control software performs the limit checking and other control guard functions to prevent running magnet support bases into each other. If the control software limit checking were to fail the clutch assemblies would prevent hardware damage should two support bases make contact.

The encoders are the optical type with 1024 steps per revolution. The encoders are also absolute, meaning that they maintain their positional information through power off-on cycles. The lead screws that are used to convert rotational motion to linear translation have a pitch of eight threads per inch. This produces a theoretical minimum translation distance of 0.00012 inch. The lead screws use zero-backlash design drive nuts. Even with this zero-backlash design the minimum translation distance is likely slightly greater than 0.00012 inch

\section{TESTING THE MECHANICAL FUNCTION OF THE MICROSCOPE}

Testing of the mechanical systems took place prior to the beam line installation. The system hardware and software performed as designed. PMQ support bases were moved in increments as small as 0.0005 to 0.001 inch with proper placement and positional tracking by the motion control hardware and software. The displacements were measured using auxiliary mechanical gages that were mounted to the lattice support plate.

\section{MECHANICAL AND IMAGING PERFORMANCE OF THE MICROSCOPE}

In October of 2002 the microscope assembly was installed onto the LANSCE Area $\mathrm{C}$ beam line. The PMQ sets were aligned to the global beam line coordinate system. Drive system communication checks were made on the microscope assembly. After the preliminary work was completed a static target was placed in the temporary test vessel up-stream from the microscope. A proton beam pulse was sent through the radiography lattice, and an image with good resolution and contrast of the static target was obtained at an approximate seven times magnification. Further tuning of the microscope resolution by slight longitudinal repositions of the PMQ sets adjusted the resolution of the static image. The inclusion of a beam collimator in the small beam tube of the microscope provided a small improvement in the resolution of the image. The microscope assembly performed well, and as designed in its first imaging experiment.

\section{CONCLUSIONS}

The ability to obtain magnified images in the approximate seven times range at heightened resolution will enable new classes of experiments to be conducted with proton radiography. Also, because the microscope magnetic lattice itself is rather compact a similar design could be used in other radiography beam lines for providing magnified images.

\section{REFERENCES}

[1]Gary Hogan, et al, "Proton Radiography", PAC99 proceedings, April, 1999.

[2]Tom Mottershead, et al, "Design and Operation of a Proton Microscope for Radiography at $800 \mathrm{MeV}$ ", these conference proceedings. 